\title{
Monotone Iterative Methods and Schwarz Methods for Nonlinear Parabolic PDE with Time Delay
}

\author{
Daoud S. Daoud \\ Department of Mathematics, Eastern Mediterranean Univ., Famaqusta, North \\ Cyprus - Mersin 10 Turkey \\ daoud.daoudQemu.edu.tr
}

\begin{abstract}
Its well known that the Schwarz alternating method proved to be feasible and powerful approach to solve elliptic or parabolic PDEs over multi overlapped subdomains. Recently Schwarz method proved to be very effective method when embedded within a well established solution methods such as monotone iterative method( or the method of lower and upper solution method). In this work we present the proofs of convergence of additive and multiplicative Schwarz alternating method for non linear parabolic equation where the reaction function involves a time delay function.
\end{abstract}

\section{Introduction}

In several applications its useful and even essential to decompose the domain of the PDE into several subdomains overlapped and non overlapped. The concept of decomposing the domain has been utilized in connections with the numerical approximation of the problem and the parallel processing algorithms. The earliest known domain decomposition method was presented by H.A. Schwarz in 1870 .

The classical alternating Schwarz method have been utilized efficiently to solve the PDE over several subdomains and then the method has gained its popularity specially after the developments of the parallel computer architecture.

Recently the method of lower and upper solution and its associated monotone iterative method have been considered to develop analytical and numerical solution algorithms. The method has found to be a powerful tool for treatment of several type of PDEs like elliptic, time dependent parabolic problem see Pao $[7,8]$, and time dependent parabolic problem which involves time delay reaction function see the 'works by X-Lu [2,3](The method is also called monotone iterative method).

On the other hand the method of lower and upper solution has also been considered with the use of the Schwarz iterative method see Lui [4]-[6], Lui studied the use of the monotone type of iterative methods in solving non linear Elliptic[4], and Parabolic equations with Schwarz methods [6]. 
The solution of the time delay problem by the lower and upper solution method and its associated monotone iterative method also has been studied numerically and analytically by Feng and $\mathrm{Lu}[1]$, and $\mathrm{Lu}[2,3]$ and the references in $\mathrm{Pao}[7]$.

In Pao [7] presented a comprehensive study and complete bibliography for the monotone iterative method and the application for the time delay problem.

This article is an extension of the analysis by Lui [6] to study the Schwarz method with monotone iteration to solve the time dependent parabolic problem where the reaction function involves a time delay function such that the considered type of problem will fulfill the analysis by the monotone method see $[7]$.

In section 3 we present some preliminaries notations and the definition of the model problem and in section 4 we present the proof for the multiplicative and the additive Schwarz method when the reaction function involves a monotone non decreasing time delay function. In section 5 we present the proofs of the Schwarz methods when the reaction function involves a monotone non increasing time delay function and the conclusion in section 6 .

\section{Preliminaries}

Consider the following type of parabolic boundary value problem where the reaction function involves a time delay function given by

$$
u_{t}-\Delta u=f(t, x, u(t, x))+g(t, x, u(t-r, x)) \text { on } D=[0, T] \times \Omega,
$$

with

$$
u(t, x)=h \text { on } S, \text { and } u(t, x)=\eta_{0}(t, x) \text { on } D_{(-r)},
$$

and $D_{(-r)}=[-r, 0] \times \Omega$ where $\Omega$ is assumed to be a bounded domain in $\mathbf{R}^{N}$. We assume that $f, g$ are Hölder continuous and $\eta_{0}(t, x)$ is also Hölder continuous on $D_{(-r)}$. We also assume that $\Omega$ is decomposed into $m, m \geq 2$, subdomains with smooth boundaries such that $\Omega=\Omega_{1} \cup \Omega_{2} \cup \ldots \cup \Omega_{m}$.

We are interested in the solution of the parabolic PDE (1) for the time interval $[-r, T]$ for some fixed positive $T$.

In this area a couple of well known examples of the equation (1) are the model problems known as the diffusive logistics equation and Fisher's diffusive equation in population genetics [7]. Define $D_{i}=(0, T) \times \Omega_{i}, S=(0, T) \times \partial \Omega, S_{i}=$ $(0, T) \times \Omega_{i}, D_{(-r)}=[-r, 0] \times \Omega$, and $D_{i,(-r)}=[-r, 0] \times \Omega_{i}$.

Let $X$ denote the space of functions in $C\left(\overline{E_{T}}\right), E_{T}=[-r, T] \times \Omega$, which are continuously differentiable in time and twice differentiable in $\Omega$, and we are interested in the solutions of PDE's in this space. The pair of functions $(\underline{u}, \bar{u})$ is said to be ordered if $\underline{u} \leq \bar{u}$ in $E_{T}$.

Let $\mathcal{A}$ be the sector of smooth functions defined by

$$
\mathcal{A}=\{u \in X: \underline{u} \leq u \leq \bar{u} \text { on } \bar{D}\} .
$$


In addition suppose there exist some non negative continuous functions $\underline{c}$, and $\bar{c}$ defined on $\bar{D}$ by

$$
\begin{aligned}
& \underline{c}(t, x)=\sup \left\{-f_{u}(t, x, u) ; \underline{u} \leq \bar{u}\right\}, \text { and } \\
& \bar{c}(t, x)=\sup \left\{f_{u}(t, x, u) ; \underline{u} \leq \bar{u}\right\}
\end{aligned}
$$

such that

$\underline{c}(u-v) \leq(f(t, x, u)+g(t, x, u(t-r, x)))-(f(t, x, v)+g(t, x, v(t-r, x))) \leq \bar{c}(u-v)$

for any $v \leq u \in \mathcal{A}$.

The function $g$ in (1) is either a monotone nondecreasing or monotone nonincreasing function in $\eta$ for $\eta$ in the sector of upper and lower solutions [7]. An essential theorems in this study are the following maximum principles theorems

Theorem 1 (Maximum Principle Theorem). Let $w \in X$ satisfy

$$
w_{t}-\Delta w+\underline{c} \geq 0 \text { on } D, w \geq 0 \text { on } S, \text { and } w(0, x) \geq 0 \text { on } \Omega .
$$

Then $w \geq 0$ on $\bar{D}$.

In cases when the functions are less smooth or the domain is with non smooth boundary a generalized maximum principle theorem is also required see [6], given by the following generalized form.

Theorem 2 (Generalized Maximum Principle). Suppose $\Psi$ is an open set in $\mathbf{R}^{N}$ and $\Psi_{T}=(-r, T) \times \Psi$, let $w \in H^{1}\left(-r, T ; H^{1}(\Psi) \cap C(\bar{\Psi})\right)$ and satisfy

$\int_{\Psi_{T}}\left(\nabla w \cdot \nabla \phi+w_{t} \phi+\underline{c} w \phi\right) \geq 0$, for non negative $\phi \in H^{1}\left(-r, T ; H^{1}(\Psi) \cap C(\bar{\Psi})\right)$ and $w(0) \geq 0$ on $\Psi$. Then $w \geq 0$ on $\overline{\Psi_{T}}$.

The study in this article is divided into two parts, the first part section 4 is dealing with the case when $g$ is a monotone nonincreasing function and the second part section 5 is dealing with the case when $g$ is a monotone nondecreasing function.

\section{Monotone Nonincreasing Function}

This section investigate the application of Schwarz methods to the parabolic PDEs (1), where the reaction function involves a monotone nonincreasing time delay function $g(t, x, u(t-r, x))$. In this case the generated sequence of lower and upper solutions are coupled pair of sequence and are defined as follows.

Definition 1. Let $g(. \eta)$ be a monotone nonincreasing in $\eta$. Then a pair of function $\underline{u}, \bar{u} \in X$ are called coupled upper and lower solutions of PDE (1), if $\bar{u} \geq \underline{u}$ in $X$, and they satisfy the inequalities;

$$
\begin{aligned}
& \bar{u}_{t}-\Delta \bar{u} \geq f(\bar{u})+g(\underline{u}(t-r)) \quad \text { and } \\
& \underline{u}_{t}-\Delta \underline{u} \leq f(\underline{u})+g(\bar{u}(t-r)) \quad \text { in } D_{T}
\end{aligned}
$$

with $\underline{u}<h<\bar{u}$ on $S$, and $\underline{u} \leq \eta_{0}(t, x) \leq \bar{u}$ on $D_{(-r)}$. 
For a given pair of coupled upper and lower solutions $\bar{u}, \underline{u}$ and using $\bar{u}^{(0)}=$ $\bar{u}$, and $\underline{u}^{(0)}=\underline{u}$ we construct two sequences of $\left\{\bar{u}^{(k)}, \underline{u}^{(k)}\right\}$ from the following iterative process

$$
\begin{aligned}
& \bar{u}_{t}^{(k)}-\Delta \bar{u}^{(k)}+\underline{c} \bar{u}^{(k)}=\underline{c} \bar{u}^{(k-1)}+f\left(\bar{u}^{(k-1)}\right)+g\left(\underline{u}^{(k-1)}\right) \\
& \underline{u}_{t}^{(k)}-\Delta \underline{u}^{(k)}+\underline{c} \underline{u}^{(k)}=\underline{c} \underline{u}^{(k-1)}+f\left(\underline{u}^{(k-1)}\right)+g\left(\bar{u}^{(k-1)}\right)
\end{aligned}
$$

subject to the following boundary and initial conditions

$$
\bar{u}^{(k)}=h, \underline{u}^{(k)}=h \text { on } S, \bar{u}^{(k)}=\eta_{0}(t, x), \quad \text { and } \underline{u}^{(k)}=\eta_{0}(t, x) \text { on } D_{(-r)} .
$$

The generated sequence of pair of solutions $\left\{\underline{u}^{(k)}, \bar{u}^{(k)}\right\}$ are well defined and monotone. Furthermore the generated sequences convergence monotically to the unique solution of (1) when $g_{u} \leq 0$ (Theorem 8.2 in [7]).

For the above definition of sequences of pair of solutions corresponding to the monotone nonincreasing function $g$ we present the convergence of sequence(pair) of solutions generated from the Multiplicative Schwarz method by the following theorem;

Theorem 3. Let $\bar{u}^{(0)}=\bar{u}^{(-1 / 2)}=\bar{u}$ and $\underline{u}^{(0)}=\underline{u}^{(-1 / 2)}=\underline{u}$ on $\bar{D}$, with $\underline{u}=$ $h$, and $\bar{u}=h$ on $S$, and $\bar{u}=\underline{u}=\eta_{0}$ on $D_{(-r)}$.

The Schwarz sequence for $n \geq 0$, is defined by

$$
\begin{aligned}
& \left(\partial_{t}-\Delta+\underline{c}\right) \bar{u}^{(n+1 / 2)}=f\left(\bar{u}^{(n-1 / 2)}\right)+\underline{c} \bar{u}^{(n-1 / 2)}+g\left(\underline{u}^{(n-1 / 2)}\right) \\
& \left(\partial_{t}-\Delta+\underline{c}\right) \underline{u}^{(n+1 / 2)}=f\left(\underline{u}^{(n-1 / 2)}\right)+\underline{c} \underline{u}^{(n-1 / 2)}+g\left(\bar{u}^{(n-1 / 2)}\right)
\end{aligned}
$$

with

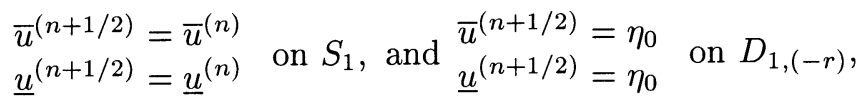

and

$$
\begin{aligned}
&\left(\partial_{t}-\Delta+\underline{c}\right) \bar{u}^{(n+1)}=f\left(\bar{u}^{(n)}\right)+\underline{c} \bar{u}^{(n)}+g\left(\underline{u}^{(n)}\right) \\
&\left(\partial_{t}-\Delta+\underline{c}\right) \underline{u}^{(n+1)}=f\left(\underline{u}^{(n)}\right)+\underline{c} \underline{u}^{(n)}+g\left(\bar{u}^{(n)}\right) \\
& \bar{u}^{(n+1)}=\bar{u}^{(n+1 / 2)} \quad \text { on } S_{2}, \text { and } \begin{array}{l}
\bar{u}^{(n+1)}=\eta_{0} \\
\underline{u}^{(n+1)}=\eta_{0}
\end{array} \quad \text { on } D_{2,(-r)} \cdot
\end{aligned}
$$

Here $\underline{u}^{(n+1 / 2)}$ and $\bar{u}^{(n+1 / 2)}$ are defined as $\underline{u}^{(n)}$ and $\bar{u}^{(n)}$ on $\bar{D} \backslash \bar{D}_{1}$ respectively, and $\underline{u}^{(n+1)}$ and $\bar{u}^{(n+1)}$ are defined as $\underline{u}^{(n+1 / 2)}$ and $\bar{u}^{(n+1 / 2)}$ on $\bar{D} \backslash \bar{D}_{2}$, respectively. Then $\underline{u}^{(n+i / 2)} \rightarrow u$ and $\bar{u}^{(n+i / 2)} \rightarrow u$ on $D_{i}, i=1,2$, where $u$ is the solution of (1) in $\mathcal{A}$.

Proof. To show the convergence of the lower and upper solution sequence to the solution $u$ on $D_{i}$, we start to prove that the pair of coupled lower and upper sequence is monotone i.e. to show that 
$\underline{u} \leq \underline{u}^{(n-1 / 2)} \leq \underline{u}^{(n)} \leq \underline{u}^{(n+1 / 2)} \leq \bar{u}^{(n+1 / 2)} \leq \bar{u}^{(n)} \leq \bar{u}^{(n-1 / 2)} \leq \bar{u}$ on $\bar{D}$,

For $n=0$, consider the sequence on $D_{1}$ for $\bar{u}^{(1 / 2)}$ and the upper solution $\bar{u}^{(0)}$

$$
\begin{gathered}
\left(\partial_{t}-\Delta+\underline{c}\right) \bar{u}^{(1 / 2)}=f\left(\bar{u}^{(0)}\right)+\underline{c} \bar{u}^{(0)}+g\left(\underline{u}^{(0)}\right) \\
\left(\partial_{t}-\Delta+\underline{c}\right) \bar{u}^{(0)} \geq f\left(\bar{u}^{(0)}\right)+\underline{c} \bar{u}^{(0)}+g\left(\underline{u}^{(0)}\right)
\end{gathered}
$$

On subtraction of the above two results we obtain

$$
\left(\partial_{t}-\Delta+\underline{c}\right)\left(\bar{u}^{(0)}-\bar{u}^{(1 / 2)}\right) \geq 0 .
$$

Since $\bar{u}^{(0)}-\bar{u}^{(1 / 2)}=0$ on $S_{1}$, and $\bar{u}^{(0)}-\bar{u}^{(1 / 2)}=\bar{u}^{(0)}-\eta_{0}(t, x) \geq 0$ on $D_{1,(-r)}$, which implies that $\bar{u}^{(0)}-\eta_{0}(0, x) \geq 0$ on $\Omega$.

Then by the maximum principle theorem 1 we conclude that $\bar{u}^{(0)} \geq \bar{u}^{(1 / 2)}$ on $\overline{D_{1}}$, which also hold on $\bar{D}$.

For the lower solution the proof is similar to the above and it follows by reversing the second inequality in $(6)$, concluding that $\underline{u}^{(0)} \leq \underline{u}^{(1 / 2)}$. To proceed in the proof of (5) it requires the proof of the following pair of couple of inequalities

$$
\begin{aligned}
& \bar{u}^{(n+1 / 2)} \leq \bar{u}^{(n-1 / 2)} \\
& \underline{u}^{(n-1 / 2)} \leq \underline{u}^{(n+1 / 2)}
\end{aligned}
$$

on $D_{1}$, and on $D_{2}$ the following inequalities

$$
\begin{gathered}
\bar{u}^{(n+1)} \leq \bar{u}^{(n)} \\
\underline{u}^{(n)} \leq \underline{u}^{(n+1)} .
\end{gathered}
$$

On the subdomain $D_{1}$ for $n=0$ and for both of the inequalities in (7) they follows from the definition.

On $D_{2}$ for $n=0$ consider the defining equation of $\bar{u}^{(1)}$

$$
\left(\partial_{t}-\Delta+\underline{c}\right) \bar{u}^{(1)}=\underline{c} \bar{u}^{(0)}+f\left(\bar{u}^{(0)}+g\left(\underline{u}^{(0)}\right),\right.
$$

and the equation for $\bar{u}^{(0)}$

$$
\left(\partial_{t}-\Delta+\underline{c}\right) \bar{u}^{(0)} \geq \underline{c} \bar{u}^{(0)}+f\left(\bar{u}^{(0)}\right)+g\left(\underline{u}^{(0)}\right) .
$$

From the subtraction the above relations we obtain;

$$
\left(\partial_{t}-\Delta+\underline{c}\right)\left(\bar{u}^{(0)}-\bar{u}^{(1)}\right) \geq 0
$$

on $D_{2}$ with $\bar{u}^{(0)}-\bar{u}^{(1)}=0$ on $S_{2}$, and $\bar{u}^{(0)}-\bar{u}^{(1)} \geq 0$ on $D_{2,(-r)}$, then by the maximum principle theorem it follows that $\bar{u}^{(0)} \geq \bar{u}^{(1)}$.

For the lower solution sequence over $D_{2}$ it follows similarly by considering the defining equations for $\underline{u}^{(1)}$ and the equation for the lower solution sequence $\underline{u}^{(0)}$. 
Assume that the inequalities $(7,8)$ are holds for $n$, to prove they holds for $n$ replaced by $(n+1)$, and we firstly consider the case of $(7)$.

Consider (3) for $\bar{u}^{(n+3 / 2)}$, and $\bar{u}^{(n+1 / 2)}$

$$
\begin{aligned}
& \left(\partial_{t}-\Delta+\underline{c}\right) \bar{u}^{(n+3 / 2)}=f\left(\bar{u}^{(n+1 / 2)}\right)+\underline{c} \bar{u}^{(n+1 / 2)}+g\left(\underline{u}^{(n+1 / 2)}\right) \\
& \left(\partial_{t}-\Delta+\underline{c}\right) \bar{u}^{(n+1 / 2)}=f\left(\bar{u}^{(n-1 / 2)}\right)+\underline{c} \bar{u}^{(n-1 / 2)}+g\left(\underline{u}^{(n-1 / 2)}\right) .
\end{aligned}
$$

On subtraction the above equalities with the consideration of the definition of $\underline{c}, g_{u} \leq 0$ and the induction assumption, it follows that;

$$
\begin{gathered}
\left(\partial_{t}-\Delta+\underline{c}\right)\left(\bar{u}^{(n+1 / 2)}-\bar{u}^{(n+3 / 2)}\right)= \\
\left(f_{u}+\underline{c}\right)\left(\bar{u}^{(n-1 / 2)}-\bar{u}^{(n+1 / 2)}\right)-g_{u}\left(\underline{u}^{(n+1 / 2)}-\underline{u}^{(n-1 / 2)}\right) \geq 0,
\end{gathered}
$$

Since $\bar{u}^{(n+3 / 2)}=\bar{u}^{(n+1)}$ on $S_{1}, \bar{u}^{(n+1)} \leq \bar{u}^{(n)}$ by induction hypothesis and $\bar{u}^{(n)}=\bar{u}^{(n+1 / 2)}$ on $S_{1}$, then it follows that $\left(\bar{u}^{(n+1 / 2)}-\bar{u}^{(n+3 / 2)}\right) \geq 0$ on $S_{1}$, and $\left(\bar{u}^{(n+1 / 2)}-\bar{u}^{(n+3 / 2)}\right)=0$ on $D_{1,(-r)}$.

Therefore by the maximum principle theorem 1 it follows that $\bar{u}^{(n+1 / 2)}$ $\bar{u}^{(n+3 / 2)} \geq 0$ on $\overline{D_{1}}$ concluding that $\bar{u}^{(n+1 / 2)} \geq \bar{u}^{(n+3 / 2)}$ on $\overline{D_{1}}$.

To prove $\underline{u}^{(n+1 / 2)} \leq \underline{u}^{(n+3 / 2)}$. Consider (3) for $\underline{u}^{(n+1 / 2)}$ and $\underline{u}^{(n+3 / 2)}$

$$
\begin{aligned}
& \left(\partial_{t}-\Delta+\underline{c}\right) \underline{u}^{(n+3 / 2)}=f\left(\underline{u}^{(n+1 / 2)}+\underline{c} \underline{u}^{(n+1 / 2)}+g\left(\bar{u}^{(n+1 / 2)}\right)\right. \\
& \left(\partial_{t}-\Delta+\underline{c} \underline{u}^{(n+1 / 2)}=f\left(\underline{u}^{(n-1 / 2)}+\underline{c} \underline{u}^{(n-1 / 2)}+g\left(\bar{u}^{(n-1 / 2)}\right),\right.\right.
\end{aligned}
$$

by subtracting the above two equations we obtain

$$
\begin{gathered}
\left(\partial_{t}-\Delta+\underline{c}\right)\left(\underline{u}^{(n+3 / 2)}-\underline{u}^{(n+1 / 2)}\right)= \\
\left(f_{u}+\underline{c}\right)\left(\underline{u}^{(n+1 / 2)}-\underline{u}^{(n-1 / 2)}\right)-g_{u}\left(\bar{u}^{(n-1 / 2)}-\bar{u}^{(n+1 / 2)}\right) \geq 0 .
\end{gathered}
$$

The last inequality follows from the induction assumptions, the definition of $\underline{c}$, and $g_{u} \leq 0$.

Since $\underline{u}^{(n+3 / 2)}=\underline{u}^{(n+1)}$ on $S_{1}, \underline{u}^{(n+1)} \geq \underline{u}^{(n)}$ by induction hypothesis, and $\underline{u}^{(n)}=\underline{u}^{(n+1 / 2)}$ on $\underline{S}_{1}$, then $\underline{u}^{(n+3 / 2)}-\underline{u}^{(n+1 / 2)} \geq 0$ on $S_{1}$, and $\underline{u}^{(n+3 / 2)}-$ $\underline{u}^{(n+1 / 2)}=0$ on $D_{1,(-r)}$, then it follows that $\underline{u}^{(n+3 / 2)}-\underline{u}^{(n+1 / 2)}=0$ on $\Omega_{1}$. By the maximum principle theorem 1 implies that $\underline{u}^{(n+3 / 2)}-\underline{u}^{(n+1 / 2)} \geq 0$ on $\overline{D_{1}}$, and then concluding that $\underline{u}^{(n+3 / 2)} \geq \underline{u}^{(n+1 / 2)}$ on $\overline{D_{1}}$.

Similarly we could prove the inequalities (8) for $D_{2}$ when $n$ replaced by $n+1$.

Then assume (5)is hold for $n$ and to prove it is true for $n$ replaced by $n+1$.

On $D_{1} \backslash D_{2}$ we have $\underline{u}^{(n+1)}=\underline{u}^{(n+1 / 2)}$ on $S_{2}$ by definition and on $D_{1} \cap D_{2}$ subtract the defining equations for $\underline{u}^{(n+1 / 2)}$ and $\underline{u}^{(n+1)}$ to obtain

$$
\left(\partial_{t}-\Delta+\underline{c}\right)\left(\underline{u}^{(n+1)}-\underline{u}^{(n+1 / 2)}\right)=\left(f_{u}+\underline{c}\right)\left(\underline{u}^{(n)}-\underline{u}^{(n-1 / 2)}\right)-g_{u}\left(\bar{u}^{(n-1 / 2)}-\bar{u}^{(n)}\right) \geq 0 .
$$

The above inequality hold by the definition of $\underline{c}, g_{u} \leq 0$, and the induction hypothesis. In case that $D_{1} \cap D_{2}$ is not smooth we can multiply the above inequality by anon negative function $\phi \in H^{1}\left(-r, T ; H_{0}^{1}\left(\Omega_{1} \cap \Omega_{2}\right)\right)$ and integrate by parts to obtain

$$
\int_{D_{1} \cap D_{2}}\left(\underline{u}^{(n+1)}-\underline{u}^{(n+1 / 2)}\right)_{t} \phi+\nabla\left(\underline{u}^{(n+1)}-\underline{u}^{(n+1 / 2)}\right) \cdot \nabla \phi+\underline{c}\left(\underline{u}^{(n+1)}-\underline{u}^{(n+1 / 2)}\right) \phi \geq 0 .
$$


On $S_{1} \cap D_{2}$ we have $\underline{u}^{(n+1)} \geq \underline{u}^{n}=\underline{u}^{(n+1 / 2)}$ by (7), and by the definition, and on $S_{2} \cap D_{1}$ we have $\underline{u}^{(n+1)}=\underline{u}^{(n+1 / 2)}$. Hence $\underline{u}^{(n+1)}-\underline{u}^{(n+1 / 2)} \geq 0$ on $D \cap\left(S_{1} \cup S_{2}\right)$, and also by definition $\underline{u}^{(n+1)}(t, x)-\underline{u}^{(n+1 / 2)}(t, x)=0$ on $D_{1,(-r)} \cap$ $D_{2,(-r)}\left(i . e . \underline{u}^{(n+1)}(0, x)-\underline{u}^{(n+1 / 2)}(0, x)=0\right.$ on $\left.\Omega_{1} \cap \Omega_{2}\right)$.

By the generalized maximum principle theorem 2 we conclude that $\underline{u}^{(n+1)} \geq$ $\underline{u}^{(n+1 / 2)}$ on $\overline{D_{1} \cap D_{2}}$. On $D_{2} \backslash D_{1}$ we have $\underline{u}^{(n+1 / 2)}=\underline{u}^{(n)} \leq \underline{u}^{(n+1)}$, then we have $\underline{u}^{(n+1)} \geq \underline{u}^{(n+1 / 2)}$ on $\bar{D}$.

Following the same way we can prove that $\bar{u}^{(n+3 / 2)} \leq \bar{u}^{(n+1)}$ on $\bar{D}$.

We proved that the sequence of solutions are bounded above and below therefore the limits are well defined and exist on $\bar{D}$, given by;

$$
\lim _{n \rightarrow \infty} \underline{u}^{(n+1 / 2)}=\lim _{n \rightarrow \infty} \bar{u}^{(n+1 / 2)}=u_{1} \text {, and } \lim _{n \rightarrow \infty} \underline{u}^{(n)}=\lim _{n \rightarrow \infty} \bar{u}^{(n)}=u_{2} .
$$

Then by theorem 8.2 in Pao [7] it follows that $u_{1}$ and $u_{2}$ satisfies the PDE on $D_{1}$ and on $D_{2}$ respectively and its easily to conclude that $u_{1}=u_{2}$ on $\bar{D}$, and that is completes the proof of the theorem.

The monotone non increasing additive Schwarz method leads to the following convergence property of the pair coupled lower and upper solution sequence, given by the following theorem.

Theorem 4. Let $\underline{u}^{(0)}=\underline{u}_{i}^{(0)}=\underline{u}$, and $\bar{u}^{(0)}=\bar{u}_{i}^{(0)}=\bar{u}$ on $\bar{D}$, for $i=1, \ldots, m$ with $\underline{u}=h$, and $\bar{u}=h$ on $S$. The additive Schwarz sequence for the pair of coupled upper and lower solutions $(n \geq 1)$ is defined by;

$$
\begin{aligned}
& \left(\partial_{t}-\Delta+\underline{c}\right) \underline{u}_{i}^{(n)}=f\left(\underline{u}_{i}^{(n-1)}\right)+\underline{c} \underline{u}_{i}^{(n-1)}+g\left(\bar{u}_{i}^{(n-1)}\right) \\
& \left(\partial_{t}-\Delta+\underline{c}\right) \bar{u}_{i}^{(n)}=f\left(\bar{u}_{i}^{(n-1)}\right)+\underline{c} \bar{u}_{i}^{(n-1)}+g\left(\underline{u}_{i}^{(n-1)}\right)
\end{aligned}
$$

subject to;

$$
\underline{u}_{i}^{(n)}=\underline{u}^{(n-1)}, \text { and } \bar{u}_{i}^{(n)}=\bar{u}^{(n-1)},
$$

on $S_{i}$, and

$$
\underline{u}_{i}^{(n)}(t, x)=\eta_{0}(t, x), \text { and } \bar{u}_{i}^{(n)}(t, x)=\eta_{0}(t, x)
$$

on $D_{i,(-r)}$.

Here $\underline{u}_{i}^{(n)}=\underline{u}^{(n-1)}$, and $\bar{u}_{i}^{(n)}=\bar{u}^{(n-1)}$, on $\bar{D} \backslash \overline{D_{i}}$, and

$$
\begin{gathered}
\underline{u}^{(n)}(t, x)=\max _{i}\left\{\underline{u}_{i}^{(n)}(t, x)\right\}, \text { and } \\
\bar{u}^{(n)}(t, x)=\min _{i}\left\{\bar{u}_{i}^{(n)}(t, x)\right\}, \text { for }(t, x) \in \bar{D} .
\end{gathered}
$$

$\underline{u}_{i}^{(n)} \rightarrow u$, and $\bar{u}_{i}^{(n)} \rightarrow u$, on $D_{i}, i=i, \ldots, m$ where $u$ is the solution of $(1)$ in $\mathcal{A}$. 
The proof of theorem 4 relies on few lemmas, we will presents the required lemmas with their proofs firstly and the proof of theorem 4 will be presented afterwards.

Lemma 1. Let $g$ be a monotone nonincreasing function given by definition 1 and let $\left\{\underline{u}^{n}, \bar{u}^{n}\right\}$ be a sequence of lower and upper solution satisfying $\underline{u}^{n} \leq$ $\underline{u}^{n+1} \leq \bar{u}^{n+1} \leq \bar{u}^{n}$ on $D_{i} \subset D$. If condition (10) in theorem 4 holds and if the following PDE

$$
\left(\partial_{t}-\Delta+\underline{c}\right) \underline{h}^{(n)}=f\left(\underline{u}^{(n-1)}\right)+\underline{c} \underline{u}^{(n-1)}+g\left(\bar{u}^{(n-1)}\right)
$$

holds on $D_{i}$, and subject to

$$
\underline{h}^{(n)}=\underline{u}^{(n)} \text { on } S_{i} \text {, and } \underline{h}^{(n)}(t, x)=\eta_{0}(t, x) \text { on } D_{i,(-r)}, \text { for } n \geq 0 .
$$

such that $\underline{u}^{(-1)}=\underline{u}^{(0)}$. Then

$$
\underline{h}^{(n+1)} \geq \underline{h}^{(n)} \text { on } D_{i}
$$

and

$$
\underline{h}^{(n)} \geq \underline{u}^{(n)} \text { on } D_{i,(-r)}
$$

Lemma 2. Let $g$ be a monotone nonincreasing function given by definition 1 and let $\left\{\underline{u}^{n}, \bar{u}^{n}\right\}$ be a sequence of lower and upper solution satisfying $\underline{u}^{n} \leq$ $\underline{u}^{n+1} \leq \bar{u}^{n+1} \leq \bar{u}^{n}$ on $D_{i} \subset D$. If condition (11) in theorem 4 holds and if the following $P D E$

$$
\left(\partial_{t}-\Delta+\underline{c}\right) \bar{h}^{(n)}=f\left(\bar{u}^{(n-1)}\right)+\bar{c} \bar{u}^{(n-1)}+g\left(\underline{u}^{(n-1)}\right)
$$

holds on $D_{i}$, and subject to

$$
\bar{h}^{(n)}=\bar{u}^{(n)} \text { on } S_{i} \text {, and } \bar{h}^{(n)}(t, x)=\eta_{0}(t, x) \text { on } D_{i,(-r)}, \text { for } n \geq 0 .
$$

such that $\bar{u}^{(-1)}=\bar{u}^{(0)}$. Then

$$
\bar{h}^{(n+1)} \geq \bar{h}^{(n)} \text { on } D_{i}
$$

and

$$
\bar{h}^{(n)} \geq \bar{u}^{(n)} \text { on } D_{i,(-r)}
$$

The other necessary lemma for the proof of theorem 4 is the following lemma.

Lemma 3. Let the hypothesis in theorem 4 hold. Then over each subdomain $D_{i} \subset D$ the following integrals satisfied, 1 -

$$
\int_{D_{i}} \nabla \underline{u}^{(n)} \cdot \nabla \phi+\underline{u}_{t}^{(n)} \phi+\underline{u}^{(n)} \phi \leq\left(\int_{D_{i}} f\left(\underline{u}^{(n-1)}\right)+\underline{c}^{(n-1)}+g\left(\bar{u}^{(n-1)}\right)\right) \phi
$$


for all nonnegative $\phi \in H^{1}\left(-r, T ; H_{0}^{1}\left(\Omega_{i}\right)\right)$.

2-

$$
\int_{D_{i}} \nabla \bar{u}^{(n)} \cdot \nabla \phi+\bar{u}_{t}^{(n)} \phi+\underline{c} \bar{u}^{(n)} \phi \geq \int_{D_{i}}\left(f\left(\bar{u}^{(n-1)}\right)+\underline{c} \bar{u}^{(n-1)}+g\left(\underline{u}^{(n-1)}\right)\right) \phi
$$

for all non negative $\phi \in H^{1}\left(-r, T ; H_{0}^{1}\left(\Omega_{i}\right) \cap C(\bar{\Omega})\right)$.

In the following we will present the proof of theorem 4 .

Proof. For the proof of theorem 4, it requires to justify the following monotone properties of the coupled pair of lower and upper solution,

$$
\begin{aligned}
& \underline{u} \leq \underline{u}_{i}^{(n)} \leq \underline{u}_{i}^{(n+1)} \leq \bar{u}_{i}^{(n+1)} \leq \bar{u}_{i}^{(n)} \leq \bar{u} \text { on } D_{i}, \\
& \underline{u} \leq \underline{u}^{(n)} \leq \underline{u}^{(n+1)} \leq \bar{u}^{(n+1)} \leq \bar{u}^{(n)} \leq \bar{u} \text { on } \bar{D},
\end{aligned}
$$

and

$$
\underline{u}^{(n)} \leq \underline{u}_{i}^{(n+1)} \leq \bar{u}_{i}^{(n+1)} \leq \bar{u}^{(n)} \text { on } \bar{D} .
$$

The proof of $(20,21)$ is by induction and it is easy to show that they holds for $n=0$ which follows from the definition.

Assume that $(20,21)$ are holds for $n$, to prove they are true for $n$ replaced by $n+1$, firstly is to show that (20) satisfies the following inequalities

$$
\underline{u} \leq \underline{u}_{i}^{(n+1)} \leq \underline{u}_{i}^{(n+2)} \leq \bar{u}_{i}^{(n+2)} \leq \bar{u}_{i}^{(n+1)} \leq \bar{u} \text { on } D_{i} .
$$

Consider the iteration given by (9) for $\underline{u}_{i}^{(n+1)}$ and $\underline{u}_{i}^{(n+2)}$, respectively

$$
\begin{aligned}
& \left(\partial_{t}-\Delta+\underline{c}\right) \underline{u}_{i}^{(n+1)}=f\left(\underline{u}_{i}^{(n)}\right)+\underline{c} \underline{u}_{i}^{(n)}+g\left(\bar{u}_{i}^{(n)}\right) \\
& \left(\partial_{t}-\Delta+\underline{c}\right) \underline{u}_{i}^{(n+2)}=f\left(\underline{u}_{i}^{(n+1)}\right)+\underline{u}_{i}^{(n+1)}+g\left(\bar{u}_{i}^{(n+1)}\right) .
\end{aligned}
$$

On subtraction of the above equations we have

$$
\left(\partial_{t}-\Delta+\underline{c}\right)\left(\underline{u}_{i}^{(n+2)}-\underline{u}_{i}^{(n+1)}\right)=\left(\underline{c}+f_{u}\right)\left(\underline{u}_{i}^{(n+1)}-\underline{u}_{i}^{(n)}\right)-g_{u}\left(\bar{u}_{i}^{(n)}-\bar{u}_{i}^{(n+1)}\right) \geq 0 .
$$

The above follows from induction assumption and $g_{u} \leq 0$.

Since $\left(\underline{u}_{i}^{(n+2)}-\underline{u}_{i}^{(n+1)}\right)=\left(\underline{u}^{(n+1)}-\underline{u}^{(n)}\right) \geq 0$ on $S_{i}$ and also we have $\left(\underline{u}_{i}^{(n+2)}-\right.$ $\left.\underline{u}_{i}^{(n+1)}\right)=0$ on $D_{i,(-r)}$.

Then $\left(\underline{u}_{i}^{(n+2)}-\underline{u}_{i}^{(n+1)}\right)=0$ on $\Omega_{i}$, and then by the maximum principle theorem concluding that $\underline{u}_{i}^{(n+2)}-\underline{u}_{i}^{(n+1)} \geq 0$ on $\overline{D_{i}}\left(\underline{u}_{i}^{(n+2)} \geq \underline{u}_{i}^{(n+1)}\right.$ on $\left.\overline{D_{i}}\right)$. Similarly we can prove $\bar{u}_{i}^{(n+1)} \geq \bar{u}_{i}^{(n+2)}$ on $\bar{D}_{i}$, then (20) holds for any $n$.

To prove (21) for $n$ replaced by $n+1$, and $(t, x) \in \bar{D}$. For the lower solution sequence there exist an integer $i, 1 \leq i \leq m$ such that $\underline{u}^{(n+1)}(t, x)=\underline{u}_{i}^{(n+1)} \leq$ $\underline{u}_{i}^{(n+2)} \leq \underline{u}^{(n+2)} \leq \bar{u}^{(n+2)}$.

The above inequalities follows by definition of $u^{(n+1)}$ and the upper and lower solution. For the upper solution sequence, there exist an integer $k, 1 \leq k \leq m$ such that

$$
\bar{u}^{(n+1)}=\bar{u}_{k}^{(n+1)} \geq \bar{u}_{k}^{(n+2)} \geq \bar{u}^{(n+1)} .
$$


Therefore

$$
\underline{u} \leq \underline{u}^{(n)} \leq \underline{u}^{(n+1)} \leq \bar{u}^{(n+1)} \leq \bar{u}^{(n)} \leq \bar{u} \text {, on } \bar{D} .
$$

The proof of inequality(22) is by induction as well. For $n=0$, the inequality follows from the definition. Assume (22) holds with $n$ replaced by $(n-1)$, i.e.

$$
\underline{u}^{(n-1)} \leq \underline{u}_{i}^{(n)} \leq \bar{u}_{i}^{(n)} \leq \bar{u}^{(n-1)} \text { on } \bar{D} \text {. }
$$

To prove it holds for $n$, i.e. $\underline{u}^{(n)} \leq \underline{u}_{i}^{(n+1)} \leq \bar{u}_{i}^{(n+1)} \leq \bar{u}^{(n)}$. Consider the first inequality of lemma 3 given by;

$$
\int_{D_{i}} \nabla \underline{u}^{(n)} \cdot \nabla \phi+\underline{u}_{t}^{(n)} \phi+\underline{u}^{(n)} \phi \leq\left(\int_{D_{i}} f\left(\underline{u}^{(n-1)}\right)+\underline{c}^{(n-1)}+g\left(\bar{u}^{(n-1)}\right)\right) \phi
$$

for all nonnegative $\phi \in H^{1}\left(-r, T ; H_{0}^{1}\left(\Omega_{i}\right)\right)$.

Next multiply the defining equation for $\underline{u}_{i}^{(n+1)}$, by a nonnegative function $\phi \in H^{1}\left(-r, T ; H_{0}^{1}\left(\Omega_{i}\right)\right)$ to obtain

$$
\int_{D_{i}} \nabla \underline{u}_{i}^{(n+1)} \cdot \nabla \phi+\left(\underline{u}_{i}^{(n+1)}\right) t \phi+\underline{c} \underline{u}_{i}^{(n+1)} \phi=\int_{\Omega_{i}}\left(f\left(\underline{u}_{i}^{(n)}\right)+\underline{c} \underline{u}_{i}^{(n)}+g\left(\bar{u}_{i}^{(n)}\right)\right) \phi
$$

Subtract (23) from the last equation to obtain;

$$
\begin{aligned}
& \int_{D_{i}} \nabla\left(\underline{u}_{i}^{(n+1)}-\underline{u}^{(n)}\right) \cdot \nabla \phi+\left(\underline{u}_{i}^{(n+1)}-\underline{u}^{(n)}\right) t \phi+\underline{c}\left(\underline{u}_{i}^{(n+1)}-\underline{u}^{(n)}\right) \phi \geq \\
& \int_{D_{i}}\left(\left(f_{u}+\underline{c}\right)\left(\underline{u}_{i}^{(n)}-\underline{u}^{(n-1)}\right)-g_{u}\left(\bar{u}_{i}^{(n)}-\bar{u}^{(n-1)}\right)\right) \phi \geq 0
\end{aligned}
$$

The last inequality follows from the induction assumption, the definition of $\underline{c}$, and $g_{u} \leq 0$.

On $S_{i}$ we have $\underline{u}_{i}^{(n+1)}=\underline{u}^{(n)}$ and on $D_{i,(-r)}$, we have $\underline{u}_{i}^{(n+1)}-\underline{u}^{(n)}=0$, therefore by the generalized maximum principle we get $\underline{u}_{i}^{(n+1)} \geq \underline{u}^{(n)}$ on $\overline{D_{i}}$ and of course this inequality is also hold on $\bar{D}$.

To prove $\bar{u}^{(n)} \geq \bar{u}_{i}^{(n+1)}$ we consider the second inequality of lemma 3 given by ;

$$
\int_{D_{i}} \nabla \bar{u}^{(n)} \cdot \nabla \phi+\bar{u}_{t}^{(n)} \phi+\underline{c} \bar{u}^{(n)} \phi \geq \int_{D_{i}} f\left(\bar{u}^{(n-1)}\right)+\underline{c} \bar{u}^{(n-1)}+g\left(\underline{u}^{(n-1)}\right)
$$

for all nonnegative $\phi \in H^{1}\left(-r, T ; H_{0}^{1}\left(\Omega_{i}\right)\right)$.

Next multiply the defining equation for $\bar{u}_{i}^{(n+1)}$, by anon negative function $\phi \in H^{1}\left(-r, T ; H_{0}^{1}\left(\Omega_{i}\right)\right)$ to obtain

$$
\int_{D_{i}} \nabla \bar{u}_{i}^{(n+1)} \cdot \nabla \phi+\left(\bar{u}_{i}^{(n+1)}\right)_{t} \phi+\underline{c} \bar{u}_{i}^{(n+1)} \phi=\int_{\Omega_{i}}\left(f\left(\bar{u}_{i}^{(n)}\right)+\underline{c} \bar{u}_{i}^{(n)}+g\left(\underline{u}_{i}^{(n)}\right)\right) \phi .
$$

Subtract the last equation from (24), we obtain

$$
\begin{aligned}
& \int_{D_{i}} \nabla\left(\bar{u}^{(n)}-\bar{u}_{i}^{(n+1)}\right) \cdot \nabla \phi+\left(\bar{u}^{(n)}-\bar{u}_{i}^{(n+1)}\right)_{t} \phi+\underline{c}\left(\bar{u}^{(n)}-\bar{u}_{i}^{(n+1)}\right) \phi \geq \\
& \int_{D_{i}}\left(\left(f_{u}+\underline{c}\right)\left(\bar{u}^{(n-1)}-\bar{u}_{i}^{(n)}\right)-g_{u}\left(\underline{u}^{(n-1)}-\underline{u}_{i}^{(n)}\right)\right) \phi \geq 0 .
\end{aligned}
$$


The above inequality follows from induction assumption, the definition of $\underline{c}$, and $g_{u} \leq 0$ Since $\bar{u}_{i}^{(n+1)}=\bar{u}^{(n)}$ on $S_{i}$, and $\bar{u}_{i}^{(n+1)}-\bar{u}^{(n)}=0$ on $D_{i,(-r)}$, therefore by the generalized maximum principle theorem 1 we obtain

$$
\bar{u}^{(n)} \geq \bar{u}_{i}^{(n+1)},
$$

on $\overline{D_{i}}$ and its also holds on $\bar{D}$.

\section{Monotone Non Decreasing Function}

Definition 2. Let $g(., \eta)$ be a monotone non decreasing function in $\eta$. A function $\underline{u} \in X$ is called a lower solution of (1), if it satisfies the inequality

$$
\underline{u}_{t}-\Delta \underline{u}-f(t, x, \underline{u})-g(t, x, \underline{u}(t-r, x)) \leq 0 \text { on } D,
$$

$\underline{u} \leq h$ on $S$, and $\underline{u}(0, x) \leq \eta_{0}(t, x)$ on $D_{(-r)}$. Similarly $\bar{u}$ is called an upper solution if it satisfies the reversed inequalities in(25).

For a given pair of ordered upper and lower solutions with $u^{(0)}=\bar{u}$ and $u^{(0)}=\underline{u}$ as two independent initial iterates for the lower and upper sequences and the respective sequences from the iteration process is given by

$$
u_{t}^{n}-\Delta u^{n}+\underline{c} u^{n}=\underline{c} u^{(n-1)}+f\left(t, x, u^{(n-1)}\right)+g\left(t, x, u^{(n-1)}(t-r, x)\right) \text { on } D,
$$

with

$$
u^{n}=h, \text { on } S, \text { and } u^{n}=\eta_{0}(t, x) \text { on } D_{-r},
$$

to produce the independent monotone sequences denoted by $\left\{\bar{u}^{(n)}\right\}$, and $\left\{\underline{u}^{(n)}\right\}$, respectively. The functions $f$ and $g$ are Hölder continuous functions, and $g_{\eta} \geq 0$, for $\eta \in<\underline{u}, \bar{u}>\in \mathcal{A}$.

The following theorem present the convergence of a multiplicative Schwarz sequences for the PDE (1) over two subdomains.

Theorem 5. Let $u^{(0)}=u^{(-1 / 2)}=\underline{u}$ on $\bar{D}$, with $\underline{u}=h$ on $S$. Define the Schwarz sequence for $(n \geq 0)$ as follows ;

$$
\begin{array}{lll}
\left(\partial_{t}-\Delta+\underline{c}\right) u^{(n+1 / 2)} & =\underline{c} u^{(n-1 / 2)}+f\left(u^{(n-1 / 2)}\right)+g\left(u^{(n-1 / 2)}(t-r)\right) & \text { on } D_{1}, \\
u^{(n+1 / 2)}= & u^{(n)} \text { on } S_{1} \\
u^{(n+1 / 2)}= & \eta_{0}(t, x) \text { on } D_{1,(-r)}
\end{array}
$$

and

$$
\begin{array}{lll}
\left(\partial_{t}-\Delta+\underline{c}\right) u^{(n+1)}= & \underline{c} u^{(n)}+f\left(u^{(n)}\right)+g\left(u^{(n)}(t-r)\right) & \text { on } D_{2} \\
u^{(n+1)}= & u^{(n+1 / 2)} \text { on } S_{2} \\
u^{(n+1)}= & \eta_{0}(t, x) \text { on } D_{2,(-r)}
\end{array}
$$

Here $u^{(n+1 / 2)}$ is defined as $u^{(n)}$ on $\bar{D} \backslash \overline{D_{1}}$ and $u^{(n+1)}$ is defined as $u^{(n+1 / 2)}$ on $\bar{D} \backslash \overline{D_{2}}$.

Then $u^{(n+i / 2)} \rightarrow u$ on $D_{i}, i=1,2$ where $u$ is the solution of (1) in $\mathcal{A}$ 
The proof outlines of theorem 5 is similar to the proof given by Lui [6] in the case of parabolic equation without time delay.

The other type of Schwarz iterative method to consider is the additive type of Schwarz iterative method which posses high parallelism than the multiplicative type. The following theorem presents the general case for m-subdomains.

Theorem 6. Let $u^{(0)}=u_{i}^{(0)}=\underline{u}$ on $\bar{D}$, for $i=1, \ldots, m$ with $\underline{u}=h$ on $S$. Define the additive Schwarz sequence by $(n \geq 1)$

$$
\left(\partial_{t}-\Delta+\underline{c}\right) u_{i}^{(n)}=f\left(u_{i}^{(n-1)}\right)+\underline{c} u_{i}^{(n-1)}+g\left(u_{i}^{(n-1)}\right) \text { on } D_{i}
$$

subject to

$$
u_{i}^{(n)}=u^{(n-1)} \text { on } S_{i}, u_{i}^{(n)}=\eta_{0}(t, x) \text { on } D_{i,(-r)}, i=1, \ldots, m .
$$

Here, $u_{i}^{(n)}$ is defined as $u^{(n-1)}$ on $\bar{D} \backslash \bar{D}_{i}$, and

$$
u^{(n)}(t, x)=\max _{1 \leq i \leq m} u_{i}^{(n)}(t, x), \quad(t, x) \in \bar{D} .
$$

Then $u_{i}^{(n)} \rightarrow u$ on $D_{i}, i=1, \ldots, m$, where $u$ is the solution of (1) in $\mathcal{A}$.

\section{Conclusion}

In this work we studied the applicability of the monotone iterative method and for the parabolic equation with reaction function involves a time delay function and we proved the convergence of the generated sequence of approximations to the exact solution.

\section{References}

1. W. Feng and X. Lu. Harmless delays for permanence in a class of population models with diffusion effects, J.Math. Anal. Appl., 206:547-566, 1997.

2. X. Lu. Monotone method and convergence acceleartion for finite difference solutions of pa problems with time delays. Numer. Methods for Part. Diff. Eqs.11:591602, 1995.

3. X. Lu. Combined methods for numerical solutions of parabolic problemwith time delays, Appl. Math. Comput. 89:213:254, 1994.

4. S. H. Lui. On monotone and Schwarz alternating method for non linear elliptic pdes. M2AN35:1-15, 2001.

5. S. H. Lui. On linear monotone and Schwarz method for nonlinear elliptic pdes. Numer Math. to appear.

6. S. H. Lui. On monotone iteration and Schwarz methods for nonlinear parabolic pdes. Numer Math. to appear.

7. C. V. Pao. Nonlinear Parabolic and Elliptic Equations, Plenum Press, New york, 1992.

8. C. V. Pao. Monotone iterations for numerical solutions of reaction-diffusionconvection equations with time delay. Numer. Methods for part. Diff Eqs. 14:339$351,1998$. 